\title{
CENTRAL EXTENSIONS OF NONSYMMETRIZABLE KAC-MOODY ALGEBRAS OVER COMMUTATIVE ALGEBRAS
}

\author{
YUN GAO
}

(Communicated by Roe W. Goodman)

\begin{abstract}
For a commutative algebra $R$ over a field $k$ of characteristic zero and a nonsymmetrizable Kac-Moody algebra $g(A)$, we prove that the Lie algebra $g_{R}(A)=R \otimes_{k} g(A)$ is centrally closed. Consequently, we get a characterization of the symmetrizability of $g(A)$ by the second homology group of the Kac-Moody algebra over Laurent polynomials. Also a presentation of $g_{R}(A)$ is given when $A$ is of nonaffine type.
\end{abstract}

\section{INTRODUCTION}

Let $k$ be a field of characteristic zero and $R$ be an associative and commutative $k$-algebra with identity. Given an $l \times l$ generalized Cartan matrix (GCM, for short) $A=\left(A_{i j}\right)$, the associated Lie algebra $g(A)$ over $k$ is called a Kac-Moody algebra and has the following standard presentation:

generators: $e_{i}, f_{i}, h_{i}, 1 \leq i \leq l$; and

relations: $\left[h_{i}, e_{j}\right]=A_{i j} e_{j},\left[h_{i}, f_{j}\right]=-A_{i j} f_{j},\left[h_{i}, h_{j}\right]=0,\left[e_{i}, f_{i}\right]=h_{i}$,

for $i \neq j,\left[e_{i}, f_{j}\right]=\left(a d e_{i}\right)^{-A_{i j}+1} e_{j}=\left(a d f_{i}\right)^{-A_{i j}+1} f_{j}=0$.

Consider the $k$-vector space $g_{R}(A)=R \otimes_{k} g(A)$ and define a Lie bracket on $g_{R}(A)$ by

$$
[a \otimes x, b \otimes y]=a b \otimes[x, y],
$$

where $a, b \in R, x, y \in g(A)$. Then $g_{R}(A)$ is a perfect Lie algebra over $k$.

Kassel [7] studied the universal central extensions of $g_{R}(A)$ when $A$ is of finite type. An elegant approach to this work, which follows Wilson [10], has been given by Moody, Rao, and Yokonuma in [9]. Some of these results are used in [3]. More generally, if $A$ is indecomposable and symmetrizable, Haddi [5] computed the second homology groups of $g_{R}(A)$ as follows:

$H_{2}\left(g_{R}(A)\right)=\Omega_{R}^{1} / d R$, if $A$ is of nonaffine type,

$H_{2}\left(g_{R}(A)\right)=\Omega_{R}^{1} / d R \oplus\left(\Omega_{R}^{1} \otimes I\right)$, if $A$ is of affine type, where $\Omega_{R}^{1} / d R$ is the module of Kähler differentials of $R$ over $k$ modulo exact forms and $I$ is the augmentation ideal of the algebra $k\left[t, t^{-1}\right]$ of Laurent polynomials which is generated by $(t-1)$.

Received by the editors April 30, 1992 and, in revised form, August 31, 1992.

1991 Mathematics Subject Classification. Primary 17B67; Secondary 17B55, 17 B60. 
In this note, we will give a presentation of $g_{R}(A)$ for a nonaffine GCM $A$ and show that $H_{2}\left(g_{R}(A)\right)=0$ when $A$ is indecomposable and nonsymmetrizable. In particular, it follows that if $A$ is an indecomposable GCM, and $R=k\left[t, t^{-1]}\right.$, then $H_{2}\left(g_{R}(A)\right) \neq 0$ if and only if $A$ is symmetrizable. This means that the second homology group of a Kac-Moody algebra over Laurent polynomials characterizes the symmetrizability of the Kac-Moody algebra.

\section{Presentations for $g_{R}(A)$}

All Lie algebras under consideration will be regarded as $k$-Lie algebras. We write $x(a)=a \otimes x$ for $a \in R, x \in g(A)$, so then $g_{R}(A)$ is a Lie algebra generated by $e_{i}(a), f_{i}(a), h_{i}(a)$ for all $a \in R, 1 \leq i \leq l$. As in [4, 6, or 8], for each tuple $\left(n_{1}, \ldots, n_{l}\right)$ of nonnegative (resp. nonpositive) integers not all zero, let $g\left(n_{1}, \ldots, n_{l}\right)$ be the subspace of $g(A)$ spanned by the elements

$$
\left[e_{i_{1}}, \ldots\left[e_{i_{m-1}}, e_{i_{m}}\right] \cdots\right] \quad\left(\text { resp. }\left[f_{i_{1}}, \ldots\left[f_{i_{m-1}}, f_{i_{m}}\right] \cdots\right]\right) \text {, }
$$

where $e_{j}$ (resp. $f_{j}$ ) occurs $\left|n_{j}\right|$ times. Also let $g(0, \ldots, 0)$ be the linear span of $h_{1}, \ldots, h_{l}$ and $g\left(n_{1}, \ldots, n_{l}\right)=0$ for any other tuple of integers. Then

$$
g(A)=\coprod_{\left(n_{1}, \ldots, n_{l}\right) \in Z^{l}} g\left(n_{1}, \ldots, n_{l}\right),
$$

which is a Lie algebra gradation of $g(A)$. Obviously, this induces a gradation of $g_{R}(A)$,

$$
g_{R}(A)=\coprod_{\left(n_{1}, \ldots, n_{l}\right) \in Z^{l}} g_{R}\left(n_{1}, \ldots, n_{l}\right),
$$

where $g_{R}\left(n_{1}, \ldots, n_{l}\right)=R \otimes g\left(n_{1}, \ldots, n_{l}\right)=\left\{x(a): x \in g\left(n_{1}, \ldots, n_{l}\right), a \in\right.$ $R\}$.

For convenience, we write $\left[x_{1},\left[x_{2}, \ldots,\left[x_{m-1}, x_{m}\right] \cdots\right]\right]=\left[x_{1}, x_{2}, \ldots\right.$, $\left.x_{m-1}, x_{m}\right]$.

Let $\bar{g}_{R}(A)$ be the Lie algebra over $k$ with the following presentation:

generators: $E_{i}(a), F_{i}(a), H_{i}(a), a \in R, 1 \leq i \leq l$;

relations:

(1) $E_{i}(\alpha a+\beta b)=\alpha E_{i}(a)+\beta E_{i}(b), \quad F_{i}(\alpha a+\beta b)=\alpha F_{i}(a)+\beta F_{i}(b)$,

$$
\begin{gathered}
{\left[H_{i}(a), E_{j}(b)\right]=A_{i j} E_{j}(a b),} \\
{\left[H_{i}(a), F_{j}(b)\right]=-A_{i j} F_{j}(a b),} \\
{\left[E_{i}(a), F_{i}(b)\right]=H_{i}(a b),} \\
{\left[E_{i}(a), E_{i}(b)\right]=0,} \\
{\left[F_{i}(a), F_{i}(b)\right]=0,} \\
{\left[E_{i_{1}}\left(a_{1}\right), \ldots\left[E_{i_{m-1}}\left(a_{m-1}\right), E_{i_{m}}\left(a_{m}\right)\right] \cdots\right]} \\
=\left[E_{i_{1}}(1), \ldots\left[E_{i_{m-1}}(1), E_{i_{m}}\left(a_{1} \cdots a_{m-1} a_{m}\right)\right] \cdots\right], \\
{\left[F_{i_{1}}\left(a_{1}\right), \ldots\left[F_{i_{m-1}}\left(a_{m-1}\right), F_{i_{m}}\left(a_{m}\right)\right] \cdots\right]} \\
=\left[F_{i_{1}}(1), \ldots\left[F_{i_{m-1}}(1), F_{i_{m}}\left(a_{1} \cdots a_{m-1} a_{m}\right)\right] \cdots\right],
\end{gathered}
$$


for all $a_{1}, \ldots, a_{m}, a, b \in R, \alpha, \beta \in k, 1 \leq i_{1}, \ldots, i_{m}, i, j \leq l$, and if $i \neq j$,

$$
\begin{gathered}
{\left[E_{i}(a), F_{j}(b)\right]=0,} \\
\left(a d E_{i}(1)\right)^{-A_{i j}+1} E_{j}(a)=0, \\
\left(a d F_{i}(1)\right)^{-A_{i j}+1} F_{j}(a)=0,
\end{gathered}
$$

where $a, b \in R, 1 \leq i \neq j \leq l$.

From the definition, it is not hard to check that $H_{i}(\alpha a+\beta b)=\alpha H_{i}(a)+$ $\beta H_{i}(b)$, and

$$
\begin{gathered}
{\left[H_{i}(a), H_{j}(b)\right]=0,} \\
{\left[H_{j}(a),\left[E_{i_{1}}(1), \ldots, E_{i_{m-1}}(1), E_{i_{m}}(b)\right]\right]} \\
=\left(A_{j i_{1}}+\cdots+A_{j i_{m}}\right)\left[E_{i_{1}}(1), \ldots, E_{i_{m-1}}(1), E_{i_{m}}(a b)\right], \\
{\left[H_{j}(a),\left[F_{i_{1}}(1), \ldots, F_{i_{m-1}}(1), F_{i_{m}}(b)\right]\right]} \\
=-\left(A_{j i_{1}}+\cdots+A_{j i_{m}}\right)\left[F_{i_{1}}(1), \ldots, F_{i_{m-1}}(1), F_{i_{m}}(a b)\right],
\end{gathered}
$$

where $a, b \in R, \alpha, \beta \in k, 1 \leq i_{1}, \ldots, i_{m}, i, j \leq l$.

One can easily see that the Lie algebra $g_{R}(A)$ satisfies the above defining relations of $\bar{g}_{R}(A)$ (replacing $E_{i}(a), F_{i}(a), H_{i}(a)$ by $e_{i}(a), f_{i}(a), h_{i}(A)$, respectively). By the universal property of $\bar{g}_{R}(A)$, there exists a homomorphism $\varphi$ from $\bar{g}_{R}(A)$ onto $g_{R}(A)$ such that

$$
\varphi\left(E_{i}(a)\right)=e_{i}(a), \quad \varphi\left(F_{i}(a)\right)=f_{i}(a), \quad \varphi\left(H_{i}(a)\right)=h_{i}(a)
$$

for all $a \in R, 1 \leq i \leq l$.

Let $\bar{g}(A)$ be the subalgebra of $\bar{g}_{R}(A)$ generated by $\left\{E_{i}(1), F_{i}(1), H_{i}(1)\right.$, $1 \leq i \leq l\}$. The restriction of $\varphi$ on $\bar{g}(A)$ induces a homomorphism from $\bar{g}(A)$ onto $g(A)$ which we shall denote by $\psi$. Since $g(A)$ has a universal property, there exists a homomorphism $\rho$ from $g(A)$ onto $\bar{g}(A)$ such that

$$
\rho\left(e_{i}\right)=E_{i}(1), \quad \rho\left(f_{i}\right)=F_{i}(1), \quad \rho\left(h_{i}\right)=H_{i}(1), \quad \text { for } 1 \leq i \leq l .
$$

It follows that $\rho \circ \psi$ is the identity map, proving that $\psi$ is an isomorphism.

Fix a basis $\left\{r_{\lambda}\right\}_{\lambda \in \Lambda}$ of $R$ with $1 \in\left\{r_{\lambda}\right\}_{\lambda \in \Lambda}$, where $\Lambda$ is an index set. Then

$$
g_{R}(A)=\coprod_{\left(n_{1}, \ldots, n_{l}\right) \in Z^{l}}\left(\coprod_{\lambda \in \Lambda} r_{\lambda} \otimes g\left(n_{1}, \ldots, n_{l}\right)\right) .
$$

Now we assume that $A$ is of nonaffine type and recall the following wellknown fact (see Theorem 4.3 in [6] or Proposition 3.6.5 in [8]).

Lemma 1. If $A=\left(A_{i j}\right)$ is an indecomposable and nonaffine $G C M$, and $n_{1}, \ldots, n_{l}$ are nonnegative integers such that

$$
\begin{gathered}
A_{11} n_{1}+\cdots+A_{1 l} n_{l}=0 \\
\vdots \\
A_{1 l} n_{1}+\cdots+A_{l l} n_{l}=0
\end{gathered}
$$

then $n_{1}=\cdots=n_{l}=0$.

To define a linear mapping from $g_{R}(A)$ to $\bar{g}_{R}(A)$, we need the following elementary result. 
Lemma 2. Suppose $V$ is any vector space over $k$ with $\left\{x_{i}\right\}_{i \in I}$ spanning $V$ and let $W$ be another vector space over $k$ with elements $\left\{y_{i}\right\}_{i \in I} \subseteq W$. If $\sum_{i} \alpha_{i} x_{i}=0$ implies $\sum_{i} \alpha_{i} y_{i}=0$, then there is a unique linear map $\phi$ from $V$ to $W$ satisfying $\phi\left(\sum_{i} \alpha_{i} x_{i}\right)=\sum_{i} \alpha_{i} y_{i}$.

We define a mapping $\phi: g_{R}(A) \rightarrow \bar{g}_{R}(A)$ given by

$$
\begin{gathered}
\phi\left(\sum_{i=1}^{l} \alpha_{i} h_{i}\left(r_{\lambda}\right)\right)=\sum_{i=1}^{l} \alpha_{i} H_{i}\left(r_{\lambda}\right), \\
\phi\left(\sum_{\sigma \in S_{m}} \alpha_{\sigma}\left[e_{i_{\sigma(1)}}(1), \ldots, e_{i_{\sigma(m-1)}}(1), e_{i_{\sigma(m)}}\left(r_{\lambda}\right)\right]\right) \\
=\sum_{\sigma \in S_{m}} \alpha_{\sigma}\left[E_{i_{\sigma(1)}}(1), \ldots, E_{i_{\sigma(m-1)}}(1), E_{i_{\sigma(m)}}\left(r_{\lambda}\right)\right], \\
\phi\left(\sum_{\sigma \in S_{m}} \alpha_{\sigma}\left[f_{i_{\sigma(1)}}(1), \ldots, f_{i_{\sigma(m-1)}}(1), f_{i_{\sigma(m)}}\left(r_{\lambda}\right)\right]\right) \\
=\sum_{\sigma \in S_{m}} \alpha_{\sigma}\left[F_{i_{\sigma(1)}}(1), \ldots, F_{i_{\sigma(m-1)}}(1), F_{i_{\sigma(m)}}\left(r_{\lambda}\right)\right],
\end{gathered}
$$

where $\alpha_{\sigma} \in k$, and $S_{m}$ is the standard permutation group on $\{1, \ldots, m\}$.

We claim that $\phi$ is a well-defined $k$-linear mapping. Note that by (15) and Lemma 2, it suffices to show that if

$$
\sum_{\sigma \in S_{m}} \alpha_{\sigma}\left[e_{i_{\sigma(1)}}(1), \ldots, e_{i_{\sigma(m-1)}}(1), e_{i_{\sigma(m)}}\left(r_{\lambda}\right)\right]=0,
$$

where $\left[e_{i_{1}}(1), \ldots, e_{i_{m-1}}(1), e_{i_{m}}\left(r_{\lambda}\right)\right] \in r_{\lambda} \otimes g\left(n_{1}, \ldots, n_{l}\right)$ and $n_{1}, \ldots, n_{l}$ are nonnegative integers not all zero, then

$$
\sum_{\sigma \in S_{m}} \alpha_{\sigma}\left[E_{i_{\sigma(1)}}(1), \ldots, E_{i_{\sigma(m-1)}}(1), E_{i_{\sigma(m)}}\left(r_{\lambda}\right)\right]=0 .
$$

Indeed, (16) gives us

$$
\sum_{\sigma \in S_{m}} \alpha_{\sigma}\left[e_{i_{\sigma(1)}}, \ldots, e_{i_{\sigma(m-1)}}, e_{i_{\sigma(m)}}\right]=0
$$

Since $\psi$ is an isomorphism from $\bar{g}(A)$ onto $g(A)$, we have

$$
\sum_{\sigma \in S_{m}} \alpha_{\sigma}\left[E_{i_{\sigma(1)}}(1), \ldots, E_{i_{\sigma(m-1)}}(1), E_{i_{\sigma(m)}}(1)\right]=0 .
$$

Let $C=\sum_{\sigma \in S_{m}} \alpha_{\sigma}\left[E_{i_{\sigma(1)}}(1), \ldots, E_{i_{\sigma(m-1)}}(1), E_{i_{\sigma(m)}}\left(r_{\lambda}\right)\right]$. Then for any $j$, by (13), we get

$$
\begin{aligned}
{\left[H_{j}(1), C\right] } & =\left[H_{j}(1), \sum_{\sigma \in S_{m}} \alpha_{\sigma}\left[E_{i_{\sigma(1)}}(1), \ldots, E_{i_{\sigma(m-1)}}(1), E_{i_{\sigma(m)}}\left(r_{\lambda}\right)\right]\right] \\
& =\sum_{\sigma \in S_{m}} \alpha_{\sigma}\left[H_{j}(1),\left[E_{i_{\sigma(1)}}(1), \ldots, E_{i_{\sigma(m-1)}}(1), E_{i_{\sigma(m)}}\left(r_{\lambda}\right)\right]\right] \\
& =\sum_{\sigma \in S_{m}} \alpha_{\sigma}\left(A_{j 1} n_{1}+\cdots+A_{j l} n_{l}\right)\left[E_{i_{\sigma(1)}}(1), \ldots, E_{\left.\left.i_{\sigma(m-1)}(1), E_{i_{\sigma(m)}}\left(r_{\lambda}\right)\right]\right]}\right. \\
& =\left(A_{j 1} n_{1}+\cdots+A_{j l} n_{l}\right) C .
\end{aligned}
$$


Also,

$$
\begin{aligned}
{\left[H_{j}(1), C\right] } & =\left[H_{j}(1), \sum_{\sigma \in S_{m}} \alpha_{\sigma}\left[E_{i_{\sigma(1)}}(1), \ldots, E_{i_{\sigma(m-1)}}(1), E_{i_{\sigma(m)}}\left(r_{\lambda}\right)\right]\right] \\
& =\sum_{\sigma \in S_{m}} \alpha_{\sigma}\left[H_{j}\left(r_{\lambda}\right),\left[E_{i_{\sigma(1)}}(1), \ldots, E_{i_{\sigma(m-1)}}(1), E_{i_{\sigma(m)}}(1)\right]\right] \\
& =\left[H_{j}\left(r_{\lambda}\right), \sum_{\sigma \in S_{m}} \alpha_{\sigma}\left[E_{i_{\sigma(1)}}(1), \ldots, E_{i_{\sigma(m-1)}}(1), E_{i_{\sigma(m)}}(1)\right]\right]=0 .
\end{aligned}
$$

Thus $C=0$ which verifies (17), for otherwise $A_{j 1} n_{1}+\cdots+A_{j l} n_{l}=0$ for all $1 \leq j \leq l$ and this contradicts Lemma 1 .

Lemma 3. Let $L_{1}$ and $L_{2}$ be two Lie algebras over $k$. Suppose $L_{1}$ is generated by $\left\{x_{i}\right\}_{i \in I}$. If $\phi: L_{1} \rightarrow L_{2}$ is a k-linear mapping and $\phi\left(\left[x_{i}, y\right]\right)=\left[\phi\left(x_{i}\right), \phi(y)\right]$ for all $i \in I, y \in L_{1}$, then $\phi$ is a Lie algebra homomorphism.

Proof. This is a routine matter.

Clearly, $g_{R}(A)$ is actually generated by $e_{i}(a), f_{i}(a), a \in R, 1 \leq i \leq l$. We will show

$$
\phi\left(\left[e_{j}(a), y\right]\right)=\left[\phi\left(e_{j}(a)\right), \phi(y)\right], \quad \phi\left(\left[f_{j}(a), y\right]\right)=\left[\phi\left(f_{j}(a)\right), \phi(y)\right]
$$

for all $a \in R, y \in g_{R}(A), 1 \leq j \leq l$.

Case 1. Let $y \in g_{R}(0, \ldots, 0)$. Using (2), we obtain

$$
\begin{aligned}
\phi\left(\left[e_{j}(a), h_{i}(b)\right]\right) & =\phi\left(-A_{i j} e_{j}(a b)\right)=-A_{i j} \phi\left(e_{j}(a b)\right) \\
& =-A_{i j} E_{j}(a b)=\left[E_{j}(a), H_{i}(b)\right]=\left[\phi\left(e_{j}(a)\right), \phi\left(h_{i}(b)\right)\right] .
\end{aligned}
$$

Hence $\phi\left(\left[e_{j}(a), y\right]\right)=\left[\phi\left(e_{j}(a)\right), \phi(y)\right]$ for all $y \in g_{R}(0, \ldots, 0)$.

Case 2. Similar to Case $1, \phi\left(\left[f_{j}(a), y\right]\right)=\left[\phi\left(f_{j}(a)\right), \phi(y)\right]$ for all $y \in$ $g_{R}(0, \ldots, 0)$.

Case 3. Using (7), we get

$$
\begin{aligned}
\phi\left(\left[e_{j}(a), e_{i}(b)\right]\right) & =\phi\left(\left[e_{j}(1), e_{i}(a b)\right]\right)=\left[E_{j}(1), E_{i}(a b)\right] \\
& =\left[E_{j}(a), E_{i}(b)\right]=\left[\phi\left(e_{j}(a)\right), \phi\left(e_{i}(b)\right)\right] .
\end{aligned}
$$

By induction on $m$, one can see

$$
\begin{aligned}
& \phi\left(\left[e_{j}(a),\left[e_{i_{1}}(1), \ldots, e_{i_{m-1}}(1), e_{i_{m}}(b)\right]\right]\right) \\
& \quad=\left[\phi\left(e_{j}(a)\right), \phi\left(\left[e_{i_{1}}(1), \ldots, e_{i_{m-1}}(1), e_{i_{m}}(b)\right]\right)\right] .
\end{aligned}
$$

Hence $\phi\left(\left[e_{j}(a), y\right]\right)=\left[\phi\left(e_{j}(a)\right), \phi(y)\right]$ for all $y \in g_{R}\left(n_{1}, \ldots, n_{l}\right)$, where $n_{1}, \ldots, n_{l}$ are nonnegative integers not all zero.

Case 4. Similar to Case $3, \phi\left(\left[f_{j}(a), y\right]\right)=\left[\phi\left(f_{j}(a)\right), \phi(y)\right]$ for all $y \in$ $g_{R}\left(n_{1}, \ldots, n_{l}\right)$, where $n_{1}, \ldots, n_{l}$ are nonpositive integers not all zero.

Case 5. Let $y \in g_{R}\left(n_{1}, \ldots, n_{l}\right)$, where $n_{1}, \ldots, n_{l}$ are nonpositive integers not all zero. When $n_{1}+\cdots+n_{l}=n=1$, using (4) and (9), we obtain

$$
\phi\left(\left[e_{j}(a), f_{i}(b)\right]\right)=\left[\phi\left(e_{j}(a)\right), \phi\left(f_{i}(b)\right)\right] \text {. }
$$

Next, if $n_{1}+\cdots+n_{l}=n \geq 2$, we have

$$
\begin{aligned}
\phi\left(\left[e_{j}(a),\left[f_{i_{1}}(1), \ldots, f_{i_{m-1}}(1), f_{i_{m}}(b)\right]\right]\right) \\
\quad=\phi\left(\left[\left[e_{j}(a), f_{i_{1}}(1)\right],\left[f_{i_{2}}(1), \ldots, f_{i_{m-1}}(1), f_{i_{m}}(b)\right]\right]\right) \\
\quad+\phi\left(\left[f_{i_{1}}(1),\left[e_{j}(a),\left[f_{i_{2}}(1), \ldots, f_{i_{m-1}}(1), f_{i_{m}}(b)\right]\right]\right]\right) .
\end{aligned}
$$


Now, using (14),

$$
\begin{aligned}
\phi\left(\left[\left[e_{j}\right.\right.\right. & \left.\left.\left.(a), f_{i_{1}}(1)\right],\left[f_{i_{2}}(1), \ldots, f_{i_{m-1}}(1), f_{i_{m}}(b)\right]\right]\right) \\
& =\phi\left(\left[\delta_{j i_{1}} h_{j}(a),\left[f_{i_{2}}(1), \ldots, f_{i_{m-1}}(1), f_{i_{m}}(b)\right]\right]\right) \\
& =-\delta_{j i_{1}}\left(A_{j i_{2}}+\cdots+A_{j i_{m}}\right) \phi\left(\left[f_{i_{2}}(1), \ldots, f_{i_{m-1}}(1), f_{i_{m}}(a b)\right]\right) \\
& \left.=-\delta_{j i_{1}}\left(A_{j i_{2}}+\cdots+A_{j i_{m}}\right)\left[F_{i_{2}}(1), \ldots, F_{i_{m-1}}(1), F_{i_{m}}(a b)\right]\right) \\
& =\delta_{j i_{1}}\left[H_{j}(a),\left[F_{i_{2}}(1), \ldots, F_{i_{m-1}}(1), F_{i_{m}}(b)\right]\right] \\
& =\left[\left[E_{j}(a), F_{i_{1}}(1)\right],\left[F_{i_{2}}(1), \ldots, F_{i_{m-1}}(1), F_{i_{m}}(b)\right]\right]
\end{aligned}
$$

By Case 2, Case 4, and induction on $n$, we get

$$
\begin{aligned}
\phi\left(\left[f_{i_{1}}(1),\left[e_{j}(a),\left[f_{i_{2}}(1), \ldots, f_{i_{m-1}}(1), f_{i_{m}}(b)\right]\right]\right]\right) \\
\quad=\left[\phi\left(f_{i_{1}}(1)\right), \phi\left(\left[e_{j}(a),\left[f_{i_{2}}(1), \ldots, f_{i_{m-1}}(1), f_{i_{m}}(b)\right]\right]\right)\right] \\
\quad=\left[F_{i_{1}}(1),\left[E_{j}(a),\left[F_{i_{2}}(1), \ldots, F_{i_{m-1}}(1), F_{i_{m}}(b)\right]\right]\right] .
\end{aligned}
$$

So, we obtain

$$
\begin{aligned}
\phi\left(\left[e_{j}(a),\left[f_{i_{1}}(1), \ldots, f_{i_{m-1}}(1), f_{i_{m}}(b)\right]\right]\right) \\
=\left[\left[E_{j}(a), F_{i_{1}}(1)\right],\left[F_{i_{2}}(1), \ldots, F_{i_{m-1}}(1), F_{i_{m}}(b)\right]\right] \\
+\left[F_{i_{1}}(1),\left[E_{j}(a),\left[F_{i_{2}}(1), \ldots, F_{i_{m-1}}(1), F_{i_{m}}(b)\right]\right]\right] \\
=\left[E_{j}(a),\left[F_{i_{1}}(1), \ldots, F_{i_{m-1}}(1), F_{i_{m}}(b)\right]\right] \\
=\left[\phi\left(e_{j}(a)\right), \phi\left(\left[f_{i_{1}}(1), \ldots, f_{i_{m-1}}(1), f_{i_{m}}(b)\right]\right)\right] .
\end{aligned}
$$

Therefore $\phi\left(\left[e_{j}(a), y\right]\right)=\left[\phi\left(e_{j}(a)\right), \phi(y)\right]$ for all $y \in g_{R}\left(n_{1}, \ldots, n_{l}\right)$ where $n_{1}, \ldots, n_{l}$ are nonpositive integers not all zero.

Case 6. Similar to Case $5, \phi\left(\left[f_{j}(a), y\right]\right)=\left[\phi\left(f_{j}(a)\right), \phi(y)\right]$ for all $y \in$ $g_{R}\left(n_{1}, \ldots, n_{l}\right)$, where $n_{1}, \ldots, n_{l}$ are nonnegative integers not all zero.

By Lemma $3, \phi$ is a homomorphism from $g_{R}(A)$ onto $\bar{g}_{R}(A)$. Clearly $\phi \circ \varphi=i d_{\bar{g}_{R}(A)}$, so $\varphi$ is injective.

Summarizing the above, we have proved

Theorem 1. If $A$ is an indecomposable and nonaffine $G C M$, then $g_{R}(A)$ is isomorphic to $\overline{\mathrm{g}}_{R}(A)$.

\section{Central extensions of $g_{R}(A)$}

Some ideas of this section are motivated by the work of Benkart and Moody [1].

Theorem 2. If $A$ is an indecomposable and nonsymmetrizable GCM, then each central extension of the Lie algebra $g_{R}(A)$ splits.

Proof. Suppose that $0 \rightarrow V \rightarrow L \stackrel{\pi}{\rightarrow} g_{R}(A) \rightarrow 0$ is a central extension of $g_{R}(A)$.

Let $\tilde{e}_{i}(a), \tilde{h}_{i}(a)$, and $\tilde{f}_{i}(a)$, for $1 \leq i \leq l$, be preimages of $e_{i}(a), h_{i}(a)$, and $f_{i}(a)$, respectively, in $L$ under $\pi$. It is sufficient to choose $\tilde{e}_{i}(a), \tilde{h}_{i}(a)$, $\tilde{f}_{i}(a)$ for $1 \leq i \leq l, a \in\left\{r_{\lambda}\right\}_{\lambda \in \Lambda}$, and then extend our choices to all $a \in R$ by linearity.

Clearly, $\left[\tilde{h}_{i}(1), \tilde{e}_{i}(a)\right]=2 \tilde{e}_{i}(a)+c_{i}(a)$ for some $c_{i}(a) \in V$. Replacing $\tilde{e}_{i}(a)$ by $\tilde{e}_{i}(a)+\frac{1}{2} c_{i}(a)$, we get

$$
\left[\tilde{h}_{i}(1), \tilde{e}_{i}(a)\right]=2 \tilde{e}_{i}(a) \text { for } 1 \leq i \leq l, a \in R .
$$


Similarly we may take $\tilde{f}_{i}(a), \tilde{h}_{i}(a)$ so that

$$
\begin{gathered}
{\left[\tilde{h}_{i}(1), \tilde{f}_{i}(a)\right]=-2 \tilde{f}_{i}(a),} \\
{\left[\tilde{e}_{i}(1), \tilde{f}_{i}(a)\right]=\tilde{h}_{i}(a)}
\end{gathered}
$$

for $1 \leq i \leq l, a \in R$.

The proof of the theorem will be carried out in steps.

Step 1. We claim

$$
\begin{gathered}
{\left[\tilde{h}_{i}(a), \tilde{e}_{j}(b)\right]=A_{i j} \tilde{e}_{j}(a b),} \\
{\left[\tilde{h}_{i}(a), \tilde{f}_{j}(b)\right]=-A_{i j} \tilde{f}_{j}(a b),} \\
{\left[\tilde{e}_{i}(a), \tilde{e}_{i}(b)\right]=0,} \\
{\left[\tilde{f}_{i}(a), \tilde{f}_{i}(b)\right]=0,}
\end{gathered}
$$

for all $a, b \in R, 1 \leq i, j \leq l$, and if $i \neq j$,

$$
\begin{gathered}
{\left[\tilde{e}_{i}(a), \tilde{f}_{j}(b)\right]=0,} \\
\left(\operatorname{ad} \tilde{e}_{i}(1)\right)^{-A_{i j}+1} \tilde{e}_{j}(a)=0, \\
\left(\operatorname{ad} \tilde{f}_{i}(1)\right)^{-A_{i j}+1} \tilde{f}_{j}(a)=0,
\end{gathered}
$$

where $a, b \in R, 1 \leq i \neq j \leq l$.

Using (18) and the Jacobi identity, we have

$$
\begin{aligned}
{\left[\tilde{h}_{i}(a), \tilde{e}_{j}(b)\right] } & =\left[\tilde{h}_{i}(a), \frac{1}{2}\left[\tilde{h}_{j}(1), \tilde{e}_{j}(b)\right]\right] \\
& =\frac{1}{2}\left[\left[\tilde{h}_{i}(a), \tilde{h}_{j}(1)\right], \tilde{e}_{j}(b)\right]+\frac{1}{2}\left[\tilde{h}_{i}(1),\left[\tilde{h}_{i}(a), \tilde{e}_{j}(b)\right]\right] .
\end{aligned}
$$

Applying the central trick, namely, $[x, y]=\left[x^{\prime}, y^{\prime}\right]$ if $\pi(x)=\pi\left(x^{\prime}\right)$, $\pi(y)=\pi\left(y^{\prime}\right)$ for $x, y, x^{\prime}, y^{\prime} \in L$, we obtain $\left[\left[\tilde{h}_{i}(a), \tilde{h}_{j}(1)\right], \tilde{e}_{j}(b)\right]=0$ and $\left[\tilde{h}_{j}(1),\left[\tilde{h}_{i}(a), \tilde{e}_{j}(b)\right]\right]=\left[\tilde{h}_{j}(1), A_{i j} \tilde{e}_{j}(a b)\right]=2 A_{i j} \tilde{e}_{j}(a b)$.

Thus $\left[\tilde{h}_{i}(a), \tilde{e}_{j}(b)\right]=A_{i j} \tilde{e}_{j}(a b)$ which gives (21). Similarly (22) holds.

Let $i \neq j$. Since $\left[\tilde{e}_{i}(a), \tilde{f}_{j}(b)\right]=c \in V$, we see that

$$
\begin{aligned}
0 & =\left[\tilde{h}_{i}(1),\left[\tilde{e}_{i}(a), \tilde{f}_{j}(b)\right]\right]=\left[\left[\tilde{h}_{i}(1), \tilde{e}_{i}(a)\right], \tilde{f}_{j}(b)\right]+\left[\tilde{e}_{i}(a),\left[\tilde{h}_{i}(1), \tilde{f}_{j}(b)\right]\right] \\
& =\left[2 \tilde{e}_{i}(a), \tilde{f}_{j}(b)\right]-\left[\tilde{e}_{i}(a), A_{i j} \tilde{f}_{j}(b)\right]=\left(2-A_{i j}\right) c
\end{aligned}
$$

which implies $c=0$ as $A_{i j} \leq 0$, so (25) follows.

To prove (23), (24), (26), and (27), we need the following formulas which follow easily from our definition and induction.

$$
\begin{aligned}
& {\left[\tilde{h}_{j}(1),\left[\tilde{e}_{i_{1}}\left(a_{1}\right), \ldots, \tilde{e}_{i_{m}}\left(a_{m}\right)\right]\right]} \\
& =\left(A_{j i_{1}}+\cdots+A_{j i_{m}}\right)\left[\tilde{e}_{i_{1}}\left(a_{1}\right), \ldots, \tilde{e}_{i_{m}}\left(a_{m}\right)\right] \\
& {\left[\tilde{h}_{j}(1),\left[\tilde{f}_{i_{1}}\left(a_{1}\right), \ldots, \tilde{f}_{i_{m}}\left(a_{m}\right)\right]\right]} \\
& =-\left(A_{j i_{1}}+\cdots+A_{j i_{m}}\right)\left[\tilde{f}_{i_{1}}\left(a_{1}\right), \ldots, \tilde{f}_{i_{m}}\left(a_{m}\right)\right],
\end{aligned}
$$

where $1 \leq j, i_{1}, \ldots, i_{m} \leq l$ and $a_{1}, \ldots, a_{m} \in R$.

Now it is easy to see that $\left[\tilde{e}_{i}(a), \tilde{e}_{i}(b)\right] \in V$, so

$$
0=\left[\tilde{h}_{i}(1),\left[\tilde{e}_{i}(a), \tilde{e}_{i}(b)\right]\right]=4\left[\tilde{e}_{i}(a), \tilde{e}_{i}(b)\right]
$$

which gives (23). Similarly we have (24). 
Also, if $i \neq j,\left(\operatorname{ad} \tilde{e}_{i}(1)\right)^{-A_{i j}+1} \tilde{e}_{j}(a)=c \in V$, then

$$
0=\left[\tilde{h}_{i}(1), c\right]=\left(2\left(-A_{i j}+1\right)+A_{i j}\right) c=\left(-A_{i j}+2\right) c
$$

which implies $c=0$ as $A_{i j} \leq 0$. Thus (26) holds and so does (27).

Step 2. We claim

$$
\begin{aligned}
& {\left[\tilde{e}_{i_{1}}\left(a_{1}\right), \ldots, \tilde{e}_{i_{m-1}}\left(a_{m-1}\right), \tilde{e}_{i_{m}}\left(a_{m}\right)\right]} \\
& \quad=\left[\tilde{e}_{i_{1}}(1), \ldots, \tilde{e}_{i_{m-1}}(1), \tilde{e}_{i_{m}}\left(a_{1} \cdots a_{m}\right)\right], \\
& {\left[\tilde{f}_{i_{1}}\left(a_{1}\right), \ldots, \tilde{f}_{i_{m-1}}\left(a_{m-1}\right), \tilde{f}_{i_{m}}\left(a_{m}\right)\right]} \\
& \quad=\left[\tilde{f}_{i_{1}}(1), \ldots, \tilde{f}_{i_{m-1}}(1), \tilde{f}_{i_{m}}\left(a_{1} \cdots a_{m}\right)\right]
\end{aligned}
$$

for all $a_{1}, \ldots, a_{m} \in R, 1 \leq i_{1}, \ldots, i_{m} \leq l$, and $m \geq 1$. have

In fact, suppose $j$ occurs $n_{j}$ times among $\left\{i_{1}, \ldots, i_{m}\right\}$. Obviously, we

$$
\left[\tilde{e}_{i_{1}}\left(a_{1}\right), \ldots, \tilde{e}_{i_{m-1}}\left(a_{m-1}\right), \tilde{e}_{i_{m}}\left(a_{m}\right)\right]-\left[\tilde{e}_{i_{1}}(1), \ldots, \tilde{e}_{i_{m-1}}(1), \tilde{e}_{i_{m}}\left(a_{1} \cdots a_{m}\right)\right]=c
$$

for some $c \in V$. Letting $\tilde{h}_{j}(1)$ act on both sides, by (28), we get

$$
\left(A_{j 1} n_{1}+\cdots+A_{j l} n_{l}\right) c=0 \text { for all } j .
$$

$A=\left(A_{i j}\right)$ is nonsymmetrizable; it is, of course, of nonaffine type, so Lemma 1 forces $c=0$. Thus (30) holds and so does (31).

Step 3. We claim

$$
\begin{gathered}
{\left[\tilde{h}_{i}(a), \tilde{h}_{j}(b)\right]=0,} \\
{\left[\tilde{e}_{i}(a), \tilde{f}_{i}(b)\right]=\tilde{h}_{i}(a b)}
\end{gathered}
$$

for all $a, b \in R, 1 \leq i, j \leq l$.

Let $\left[\tilde{h}_{i}(a), \tilde{h}_{j}(b)\right]=H_{i j}(a, b)$, evidently $H_{i j}(a, b) \in V$, and

$$
H_{i j}(a, b)+H_{j i}(b, a)=0 .
$$

By (20), (21), and the Jacobi identity, one has

$$
\begin{aligned}
{\left[\tilde{e}_{i}(a), \tilde{f}_{i}(b)\right] } & =\left[\frac{1}{2}\left[\tilde{h}_{i}(a), \tilde{e}_{i}(1)\right], \tilde{f}_{i}(b)\right] \\
& =\frac{1}{2}\left[\tilde{h}_{i}(a),\left[\tilde{e}_{i}(1), \tilde{f}_{i}(b)\right]\right]-\frac{1}{2}\left[\tilde{e}_{i}(1),\left[\tilde{h}_{i}(a), \tilde{f}_{i}(b)\right]\right] \\
& =\frac{1}{2}\left[\tilde{h}_{i}(a), \tilde{h}_{j}(b)\right]+\left[\tilde{e}_{i}(1), \tilde{f}_{i}(a b)\right]=\tilde{h}_{i}(a b)+\frac{1}{2} H_{i i}(a, b) .
\end{aligned}
$$

From (35), we find

$$
\begin{aligned}
H_{i j}(c, a b) & =\left[\tilde{h}_{i}(c), \tilde{h}_{j}(a b)\right]=\left[\tilde{h}_{i}(c),\left[\tilde{e}_{j}(a), \tilde{f}_{j}(b)\right]\right] \\
& =\left[\left[\tilde{h}_{i}(c), \tilde{e}_{j}(a)\right], \tilde{f}_{j}(b)\right]+\left[\tilde{e}_{j}(a),\left[\tilde{h}_{i}(c), \tilde{f}_{j}(b)\right]\right] \\
& =A_{i j}\left[\tilde{e}_{j}(c a), \tilde{f}_{j}(b)\right]-A_{i j}\left[\tilde{e}_{j}(a), \tilde{f}_{j}(c b)\right] \\
& =A_{i j}\left(\tilde{h}_{j}(a b c)+\frac{1}{2} H_{j j}(c a, b)\right)-A_{i j}\left(\tilde{h}_{j}(a b c)+\frac{1}{2} H_{j j}(a, c b)\right) \\
& =\frac{1}{2} A_{i j} H_{j j}(c a, b)-\frac{1}{2} A_{i j} H_{j j}(a, c b) .
\end{aligned}
$$


In (36), setting $c=1, b=1, i=j$ we get

$$
H_{i i}(1, a)=0 \text {, }
$$

and setting $a=1$ and using (37), we have

$$
H_{i j}(c, b)=\frac{1}{2} A_{i j} H_{j j}(c, b) \text {. }
$$

Also, using (34) twice,

$$
H_{i j}(c, b)=-H_{j i}(b, c)=-\frac{1}{2} A_{j i} H_{i i}(b, c)=\frac{1}{2} A_{j i} H_{i i}(c, b) .
$$

Therefore

$$
A_{i j} H_{j j}(c, b)=A_{j i} H_{i i}(c, b) \text { for all } b, c \in R, 1 \leq i, j \leq l .
$$

Suppose that $H_{j_{0} j_{0}}(c, b) \neq 0$ for some $j_{0}$ and some $c, b \in R$. Since $A=$ $\left(A_{i j}\right)$ is indecomposable, for any $j$, there exist $j_{1}, \ldots, j_{m}$ where $j_{m}=j$ so that $A_{j_{0} j_{1}} A_{j_{1} j_{2}} \cdots A_{j_{m-1} j_{m}} \neq 0$. Iterating (39), we get $H_{j j}(c, b)=\varepsilon_{j} H_{j_{0} j_{0}}(c, b)$ for some $\varepsilon_{j} \in k, \varepsilon_{j} \neq 0$. Substitution into (39) yields $A_{i j} \varepsilon_{j} H_{j_{0} j_{0}}(c, b)=$ $A_{j i} \varepsilon_{i} H_{j_{0} j_{0}}(c, b)$. As $H_{j_{0} j_{0}}(c, b) \neq 0$ it follows that $A_{i j} \varepsilon_{j}=A_{j i} \varepsilon_{i}$. This contradicts the fact that $A$ is nonsymmetrizable.

We have shown that $H_{j j}(c, b)=0$ for all $c, b \in R, 1 \leq j \leq l$. Then (32) follows from (38) and (33) follows from (35).

Step 4. By Theorem 1, there exists a unique homomorphism $\tau$ from $g_{R}(A)$ to $L$ so that $\tau\left(e_{i}(a)\right)=\tilde{e}_{i}(a), \tau\left(f_{i}(a)\right)=\tilde{f}_{i}(a)$, and $\tau\left(h_{i}(a)\right)=\tilde{h}_{i}(a)$. Evidently, $\pi \circ \tau=i d_{g_{R}(A)}$ which says that the original sequence splits.

Combining Theorem 2 with Haddi's result, we immediately have

Corollary 1. If $A$ is an indecomposable $G C M$, and if $R$ satisfies $\Omega_{R}^{1} \neq d R$ (for instance, $\left.R=k\left[t, t^{-1}\right]\right)$, then $H_{2}\left(g_{R}(A)\right) \neq 0$ if and only if $A$ is symmetrizable.

\section{REMARKS}

Remark 1. Theorem 1 has already been worked out by Kassel [7] when $A$ is of finite type. Our proof is more direct.

Remark 2. By further analyzing the proof of Theorem 2, letting $i=j$ in (36), we get

$$
H_{i i}(c, a b)=H_{i i}(c a, b)-H_{i i}(a, c b) .
$$

From (34) and (37), we have

$$
\begin{gathered}
H_{i i}(1, a)=H_{i i}(a, 1)=0, \\
H_{i i}(a, b)+H_{i i}(b, a)=0, \\
H_{i i}(c, a b)+H_{i i}(a, b c)+H_{i i}(b, c a)=0 .
\end{gathered}
$$

We thus get another proof for the fact that $H_{2}\left(g_{R}(A)\right)=\Omega_{R}^{1} / d R$ when $A$ is an indecomposable and symmetrizable nonaffine GCM.

Remark 3. If the Lie algebra $g(A)$ is replaced by the Lie algebra $\hat{g}(A)$ which is defined by the relations: $\left[h_{i}, h_{j}\right]=0,\left[h_{i}, e_{j}\right]=A_{i j} e_{j},\left[h_{i}, f_{j}\right]=-A_{i j} f_{j}$, $\left[e_{i}, f_{j}\right]=\delta_{i j} h_{j}$, see $[2,6]$, then our methods also yield that $R \otimes_{k} \hat{g}(A)$ is centrally closed when $A$ is indecomposable and nonsymmetrizable. Also, $H_{2}\left(R \otimes_{k} \hat{g}(A)\right)=\Omega_{R}^{1} / d R$ if $A$ is an indecomposable and symmetrizable nonaffine GCM. 


\section{ACKNOWLEDGMENT}

I would like to express my sincere gratitude to my supervisor, Professor S. Berman, for his constantly stimulating advice. Also, my thanks go to $\mathrm{Y}$. Krylyuk for his helpful conversations.

\section{REFERENCES}

1. G. M. Benkart and R. V. Moody, Derivations, central extensions and affine Lie algebras, Algebras Groups Geom. 3 (1986), 456-492.

2. S. Berman, Isomorphisms and automorphisms of universal Heffalump Lie Algebras, Proc. Amer. Math. Soc. 65 (1977), 29-34.

3. S. Berman and R. V. Moody, Lie algebras graded by finite root systems and the intersection matrix algebras of Slodowy, Invent. Math. 108 (1992), 323-347.

4. H. Garland, The arithmetic theory of loop groups, Inst. Hautes Études Sci. Publ. Math. 52 (1980), 5-136.

5. A. Haddi, Détermination des extensions centrales des algébres de Kac-Moody, C. R. Acad. Sci. Paris Sér. I Math. 306 (1988), 691-694.

6. V. G. Kac, Infinite dimensional Lie algebras, Cambridge Univ. Press, London and New York, 1990.

7. C. Kassel, Kähler differentials and coverings of complex simple Lie algebras extended over a commutative algebra, J. Pure Appl. Algebra 34 (1984), 265-275.

8. R. V. Moody and A. Pianzola, Lie algebras with triangular decompositions, Wiley, New York (to appear).

9. R. V. Moody, S. Eswara Rao, and T. Yokonuma, Toroidal Lie algebras and vertex representations, Geom. Dedicata 35 (1990), 283-307.

10. R. Wilson, Euclidean Lie algebras are universal central extensions, Lie algebras and related topics, Lecture Notes in Math., vol. 933, Springer-Verlag, Berlin and New York, 1982, pp. 210-213.

Department of Mathematics, University of Saskatchewan, Saskatoon, Saskatchewan, CANADA S7N 0W0

E-mail address: gao@snoopy.usask.ca 